\title{
Prevention of thiopurine-induced early leukopenia in a Korean pediatric patient with Crohn's disease who turned out to possess homozygous mutations in NUDT15 R139C
}

\author{
Jaewoan Bae ${ }^{1}$, Byung-Ho Choe ${ }^{1,2}$, Ben Kang ${ }^{1,2}$ \\ ${ }^{1}$ Department of Pediatrics, School of Medicine, Kyungpook National University, Daegu, Korea \\ ${ }^{2}$ Crohn's and Colitis Association in Daegu-Gyeongbuk (CCAiD), Daegu, Korea
}

Received: March 16, 2020

Revised: April 30, 2020

Accepted: May 7, 2020

Corresponding author:

Ben Kang

Department of Pediatrics, School of Medicine, Kyungpook National University, 680 Gukchaebosang-ro, Jung-gu, Daegu 41944, Korea

Tel: +82-53-200-2749

Fax: $+82-53-200-2029$

E-mail: benkang@knu.ac.kr
Homozygous mutations in NUDT15 R139C are known as the major factor associated with thiopurine-induced early leukopenia, particularly in Asian patients. Therefore, NUDT15 genotyping is currently recommended before thiopurine treatment to identify patients who are NUDT15 poor metabolizers and consider the use of an alternative immunomodulatory therapy. We report a case of a 12-year-old Korean girl with Crohn's disease (CD), in whom thiopurine-induced leukopenia was prevented by initiation of azathioprine (AZA) therapy at a low dose $(0.5 \mathrm{mg} / \mathrm{kg} / \mathrm{day})$ and early detection of significant hair loss and white blood cell (WBC) count decrease at 17 days from the start of AZA treatment. The WBC count dropped from 8,970/ $\mu \mathrm{L}$ to $3,370 / \mu \mathrm{L}$ in 2 weeks, and $A Z A$ treatment was stopped because of concerns of potential leukopenia in the near future.

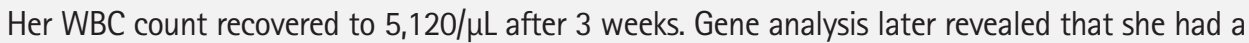
homozygous mutation in NUDT15 R139C, resulting in a poor metabolizing activity of NUDT15. In situations when NUDT15 genotyping is unavailable, initiation of AZA therapy at $0.5 \mathrm{mg} / \mathrm{kg} / \mathrm{day}$ with close observation of hair loss and WBC counts within 2 weeks may be an alternative way to prevent thiopurine-induced early leukopenia in Asian children with CD.

Keywords: Alopecia; Azathioprine; Inflammatory bowel disease; Leukopenia; NUDT15

\section{Introduction}

Crohn's disease $(\mathrm{CD})$ is a chronic inflammatory bowel disease, which can affect the entire gastrointestinal (GI) tract [1]. Approximately $25 \%$ of patients with $\mathrm{CD}$ are diagnosed $<20$ years; moreover, the incidence of $\mathrm{CD}$ has notably increased in children [1-4]. Pediatric-onset $\mathrm{CD}$ is known to have a more aggressive disease course than that of adult-onset $\mathrm{CD}$, requiring an early introduction of immunomodulators and/or anti-tumor necrosis factor (anti-TNF) agents $[5,6]$.

Thiopurines, namely azathioprine (AZA) and 6-mercaptopurine (MP), are among the most widely used immunomodulators for the treatment of pediatric $\mathrm{CD}[7]$. They are usually used as a monotherapy or combination therapy with an anti-TNF agent [7]. However, thiopurine-related adverse events, such as leukopenia, have been frequently reported in patients with $\mathrm{CD}$, particularly in Asian patients [8,9]. Moreover, some patients develop severe life-threatening leukopenia during early thiopurine therapy [9-11]. Although mutations in the TPMT gene has been considered the major factor associated with leukopenia in Western countries, the life-threatening thiopurine-induced early leukopenia could not be explained by TPMT gene polymorphisms alone in Korean patients because the incidence of TPMT variants in the Korean population is lower than that in the Caucasian populations [12]. How-

Copyright (C) 2020 Yeungnam University College of Medicine

This is an Open Access article distributed under the terms of the Creative Commons Attribution Non-Commercial License (http://creativecommons.org/licenses/by-nc/4.0/) which permits unrestricted non-commercial use, distribution, and reproduction in any medium, provided the original work is properly cited. 
ever, a recent genome-wide association study has shown that thiopurine-induced early leukopenia is strongly associated with a common missense variant of the NUDT15 gene encoding R139C in Koreans [10]. Therefore, NUDT15 genotyping is recommended before thiopurine treatment to identify patients susceptible to thiopurine-induced early leukopenia, particularly in Asians [13]. However, its application in real life is still limited owing to its high costs.

We report a case of a 12-year-old girl with $\mathrm{CD}$, in whom thiopurine-induced early leukopenia was prevented by starting AZA at a low dose followed by discontinuation of AZA owing to significant hair loss and an extensive decrease in WBC count at 2 weeks after treatment. Genotyping later revealed that she had a homozygous mutation in NUDT15 R139C.

\section{Case}

This study was approved by the Institutional Review Board (IRB) of Kyungpook National University Chilgok Hospital (IRB No: 2020-05-001).

A 12-year-old Korean girl was admitted due to abdominal pain and diarrhea for 3 months. She also had weight loss of $8 \mathrm{~kg}$ during this period without any decrease in her growth velocity. Past medical history of the patient and family was unremarkable.

On admission, her vital signs were stable and within normal limits. Abdominal tenderness without rebound tenderness was evident in the right lower quadrant. An anal fissure and perianal skin tag were also observed on physical examination. Her sexual maturity was Tanner stage 4. Initial laboratory tests showed a WBC count $10,680 / \mu \mathrm{L}$, hemoglobin $11.1 \mathrm{~g} / \mathrm{dL}$, platelet count $694,000 /$ $\mu \mathrm{L}$, erythrocyte sedimentation rate (ESR) $77 \mathrm{~mm} / \mathrm{hr}$, C-reactive protein (CRP) $8.3 \mathrm{mg} / \mathrm{dL}$, and albumin $3 \mathrm{~g} / \mathrm{dL}$. Stool occult blood test was positive, and fecal calprotectin level was $>2,000 \mu \mathrm{g} /$ g. No pathogens were detected on stool culture and stool polymerase chain reaction (PCR). Ileocolonoscopy revealed skipped lesions of small to very large ulcers from the terminal ileum to the rectum (Fig. 1). Small aphthous ulcers were also observed in the esophagus and stomach on upper GI endoscopy. Cryptitis and crypt abscesses were observed throughout the terminal ileum and colon on histology; however, the acid-fast bacillus smear and culture, as well as PCR for tuberculosis were negative. Magnetic resonance enterography (MRE) revealed multiple skipped lesions throughout the ileum and colon without evidence of stenosing or fistulizing complications in the abdomen. Supplementary pelvic magnetic resonance imaging conducted with the MRE showed T2 hyperintensity at the 1 o'clock and 12 o'clock intersphincteric area without evident tract-like structures. Chest radiography showed no abnormal findings in the lungs, and the interferon-gamma release assay was negative. The patient was diagnosed with $C D$ with a phenotype of A1b, L3+L4ab, B1, G0 according to the Paris classification. Her pediatric Crohn's disease activity index (PCDAI) score was 60, and simple endoscopic score for CD (SES-CD) was 31.

Treatment was started with exclusive enteral nutrition (EEN), mesalazine, and AZA. AZA was initiated at a dose of $17 \mathrm{mg} /$ day $(0.5 \mathrm{mg} / \mathrm{kg} /$ day $)$. The patient was discharged 10 days after admission. Her laboratory examinations conducted on the day of discharge showed a WBC count $8,970 / \mu \mathrm{L}$, hemoglobin $9.7 \mathrm{~g} / \mathrm{dL}$, platelet count 584,000/ $\mathrm{LL}$, ESR $65 \mathrm{~mm} / \mathrm{hr}, \mathrm{CRP} 0.1 \mathrm{mg} / \mathrm{dL}$, and albumin $3.2 \mathrm{~g} / \mathrm{dL}$. Her PCDAI score was 40.

A follow-up was conducted at the outpatient clinic 2 weeks after discharge. Her laboratory exams revealed a WBC count 3,370/ $\mu \mathrm{L}$,
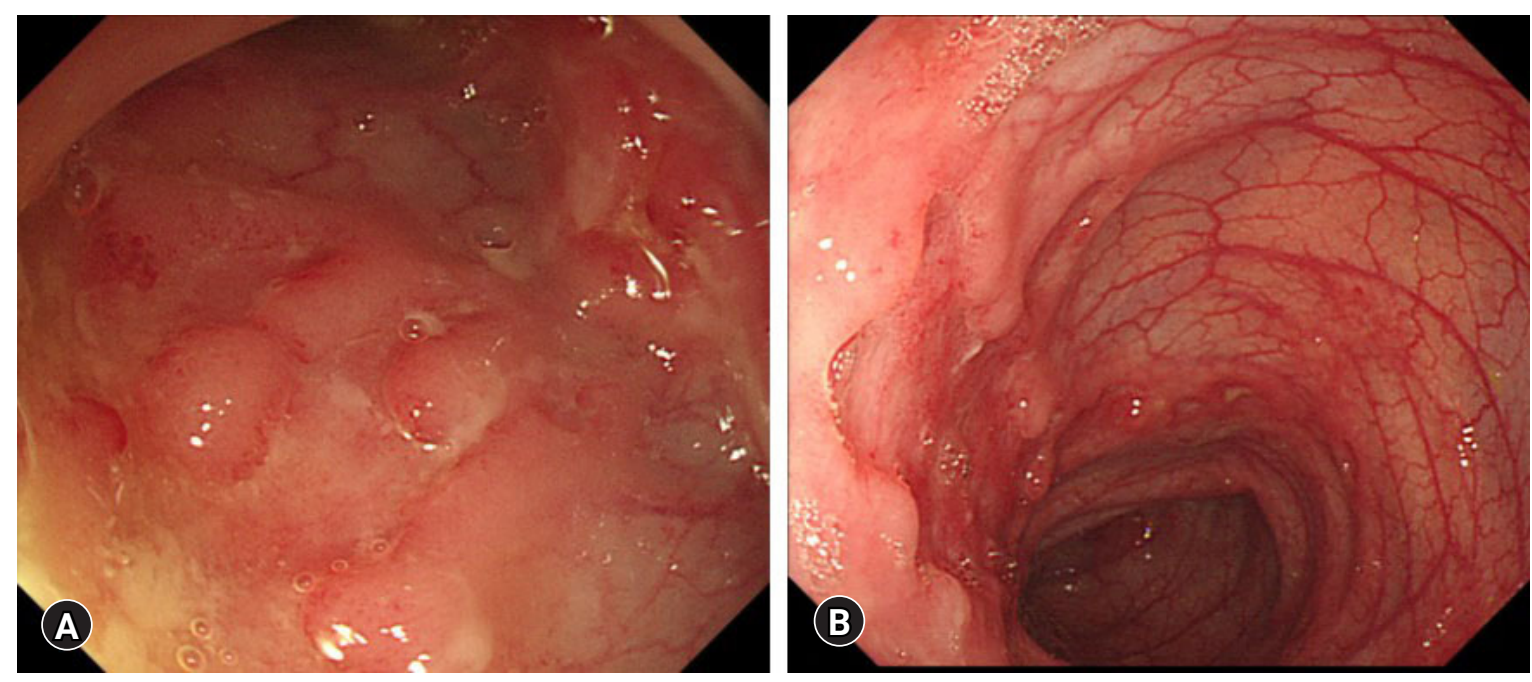

Fig. 1. Endoscopic images at diagnosis. Multiple ulcers of various sizes are noted in the terminal ileum (A) and colon (B). 
hemoglobin $10.2 \mathrm{~g} / \mathrm{dL}$, platelet count 402,000/ $\mu \mathrm{L}$, ESR $11 \mathrm{~mm} /$ $\mathrm{hr}, \mathrm{CRP} 0.04 \mathrm{mg} / \mathrm{dL}$, and albumin $4.1 \mathrm{~g} / \mathrm{dL}$. Her GI symptoms improved, and PCDAI score was 15 . However, she complained of significant hair loss. Owing to the hair loss and extensive decrease in WBC counts, AZA was discontinued because of concerns of potential thiopurine-induced leukopenia in the near future. The results of TPMT and NUDT15 gene analysis conducted during her first admission were pending.

After 3 weeks, her laboratory examinations showed a WBC count 5,120/ $\mu \mathrm{L}$, hemoglobin $12.7 \mathrm{~g} / \mathrm{dL}$, platelet count $285,000 / \mu \mathrm{L}$, ESR 5 $\mathrm{mm} / \mathrm{hr}, \mathrm{CRP} 0.01 \mathrm{mg} / \mathrm{dL}$, and albumin $4.4 \mathrm{~g} / \mathrm{dL}$ (Fig. 2). Her PCDAI score was 0 , and her hair loss improved. TPMT and NUDT15 gene analysis revealed TPMT ${ }^{*} 1 /{ }^{*} 1$ genotype (normal metabolizer) and a homozygous mutation in NUDT15 R139C comprising a diplotype of NUDT15 ${ }^{*} 3{ }^{*} 3$ (poor metabolizer), respectively.

EEN was continued for 2 additional weeks (a total of 8 weeks). Oral methotrexate was started at a dose of $12.5 \mathrm{mg} /$ week as an alternative immunomodulatory therapy to AZA. She maintained clinical remission $(\mathrm{PCDAI}<10)$ with methotrexate and mesalazine treatment. However, after 4 months, her symptoms relapsed, and infliximab was started. Currently, she is maintaining clinical remission with infliximab, methotrexate, and mesalazine for more than 1 year, and ileocolonoscopy performed 1 year after infliximab initiation revealed mucosal healing (SES-CD, 0).

\section{Discussion}

In this case, we were able to observe that thiopurine-induced early

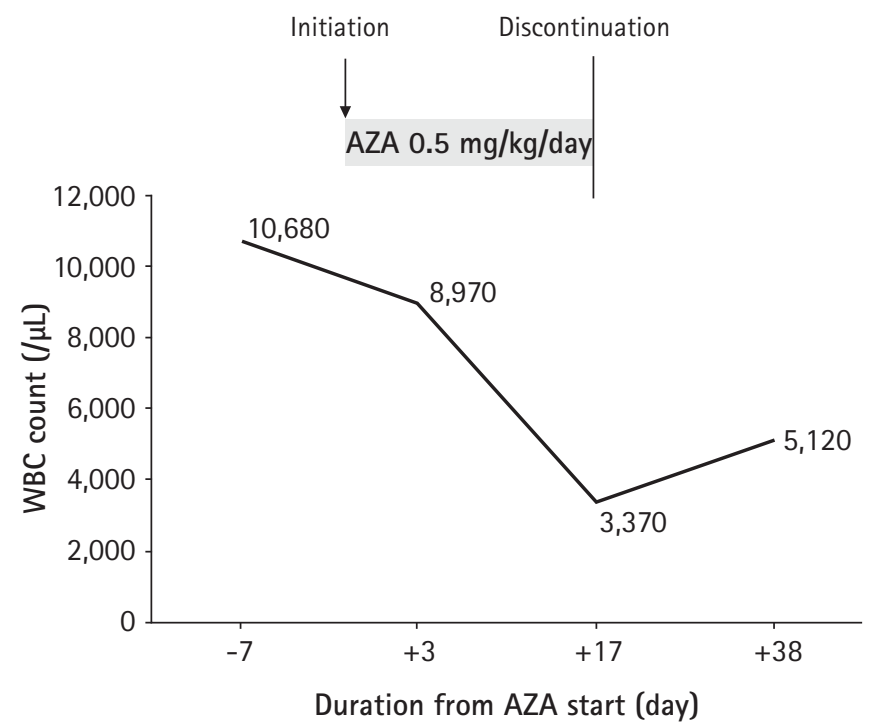

Fig. 2. Changes in white blood cell counts after the initiation and discontinuation of azathioprine in the patient. AZA, azathioprine; WBC, white blood cell. leukopenia could be prevented in patients with homozygous mutations in NUDT15 R139C (NUDT15 poor metabolizers) without knowledge of the results for NUDT15 gene analysis by starting AZA therapy at low doses with short-term monitoring of WBC counts and close observation of the associated symptoms, such as hair loss.

Thiopurines are among the most commonly used immunomodulators for the treatment of pediatric $\mathrm{CD}$ [7]. The recommended doses of AZA and 6-MP for the treatment of pediatric $\mathrm{CD}$ are $2-2.5 \mathrm{mg} / \mathrm{kg} /$ day and $1-1.5 \mathrm{mg} / \mathrm{kg} /$ day, respectively, in patients with normal TPMT metabolism [7]. Although treatment is usually started at these doses in Western countries, adult gastroenterologists in Asian countries tend to use a dose-escalating strategy by starting AZA therapy at low doses of $0.5-1 \mathrm{mg} / \mathrm{kg} / \mathrm{day}$ and then increasing the dose gradually to the target dose along with close monitoring of the laboratory results and potential adverse events in 1-2-week intervals during the first 2 months of treatment [13-15]. This difference in AZA dosing regimens in real-life practice in Asian countries is mainly attributed to the high incidence of thiopurine-induced leukopenia in Asian patients even at lower doses than those used in Western countries $[8,10]$. Although this thiopurine dose-escalating strategy is popular among adult gastroenterologists in Asia, many pediatric gastroenterologists in Asia still prescribe AZA therapy at initial doses of $2-2.5 \mathrm{mg} / \mathrm{kg} / \mathrm{day}$, which are associated with a high incidence of thiopurine-induced leukopenia.

The high incidence of this life-threatening adverse event in Asian populations cannot be explained by variants in the TPMT gene, the major genetic factor associated with thiopurine-induced leukopenia in Caucasian populations, because the frequency of TPMT mutations in Asians (1\%-3\%) is lower than that in Caucasians (10\%) [16]. Moreover, Yang et al. [10] showed a variant in the NUDT15 gene, R139C, was the major factor associated with thiopurine-induced early leukopenia in Korean patients. This NUDT15 variant showed a strong association with thiopurine-induced early leukopenia ( $<8$ weeks after treatment) in Koreans with an odds ratio of 35.6 [10]. Further studies have shown that the frequency of NUDT15 mutations is extremely rare in European populations, where $0.5 \%$ and $0 \%$ are NUDT 15 intermediate and poor metabolizers, respectively, whereas in Asian populations, $22 \%-28 \%$ and $2 \%-3 \%$ are NUDT 15 intermediate and poor metabolizers, respectively $[17,18]$. Moreover, early leukopenia of grade 3 (WBC count, $1,000-1,999 / \mu \mathrm{L}$ ) or grade 4 (WBC count $<1,000 / \mu \mathrm{L}$ ) was observed in patients with homozygous NUDT15 R139C mutations even at low initial AZA doses (0.5-1 $\mathrm{mg} / \mathrm{kg}$ /day) within 8 weeks after initiation of AZA treatment $[14,15]$. Therefore, NUDT15 genotyping is currently recommended before thiopurine treatment to identify patients who are 
NUDT15 poor metabolizers and consider the use of an alternative immunomodulatory therapy in those susceptible to thiopurine-induced early leukopenia [19]. For NUDT15 intermediate metabolizers, it is recommended to start with AZA doses of $0.6-2.4 \mathrm{mg} /$ $\mathrm{kg} /$ day, and adjust the doses based on the degree of myelosuppression [19].

However, the application of NUDT15 genotyping in real-life practice is limited mainly owing to its high cost. Additionally, the results of NUDT15 genotyping might be still unavailable at the time of thiopurine therapy initiation, as in the current case. We have been conducting both NUDT15 and TPMT genotyping in patients who are scheduled for AZA treatment at our center since 2017. However, it usually takes approximately 1 month for the test results to come out. The case reported here presented with severe disease activity with a PCDAI score, 60 and SES-CD, 31; therefore, AZA therapy was initiated at a dose of $0.5 \mathrm{mg} / \mathrm{kg} /$ day on the day after diagnosis along with EEN without waiting for the NUDT15 genotyping results. A surprisingly massive decrease in WBC count (by $6,600 / \mu \mathrm{L}$ ) within a 2-week treatment period with AZA at $0.5 \mathrm{mg} /$ $\mathrm{kg}$ /day was observed. Similarly, Asada et al. [14] reported a median decrease in WBC count by 4,550/ $\mu \mathrm{L}$ within 2 weeks of AZA treatment at doses of $0.75 \mathrm{mg} / \mathrm{kg} /$ day in NUDT 15 poor metabolizers. Hair loss was another clue that the extensive decrease in WBC count was associated with AZA treatment. Alopecia is commonly observed prior to thiopurine-induced leukopenia in Asian patients $[11,14,15,20]$. Therefore, the presence of both an extensive decrease in WBC count within 2 weeks after thiopurine therapy initiation and significant hair loss could be an indicator for thiopurine-induced early leukopenia in patients with $\mathrm{CD}$.

In conclusion, we report a case of a 12-year-old Korean girl with $\mathrm{CD}$, in whom thiopurine-induced leukopenia was prevented by initiation of AZA therapy at a low dose $(0.5 \mathrm{mg} / \mathrm{kg} /$ day $)$ followed by discontinuation of AZA owing to significant hair loss and an extensive decrease in WBC count at 2 weeks after treatment. Genotyping later revealed that she had a homozygous mutation in NUDT15 R139C (NUDT15 poor metabolizer). Pediatric gastroenterologists should keep in mind that in situations when NUDT15 genotyping is unavailable, initiation of AZA therapy at $0.5 \mathrm{mg} / \mathrm{kg} /$ day with close monitoring of hair loss and WBC counts within 2 weeks may be an alternative way to prevent thiopurine-induced early leukopenia in Asian children with CD.

\section{Acknowledgments}

\section{Conflicts of interest}

No potential conflict of interest relevant to this article was reported.

\section{Funding}

This work was supported by the National Research Foundation of Korea (NRF) grant funded by the Korean government (MSIT) (No. 2017R1C1B5076980).

\section{Author contributions}

Conceptualization, Data curation: all authors; Formal analysis: BHC, BK; Investigation: JB, BK; Supervision: BHC, BK; Writing-original draft: JB; Writing-review \& editing: $\mathrm{BHC}, \mathrm{BK}$.

\section{ORCID}

Jaewoan Bae, https://orcid.org/0000-0003-3607-2148

Byung-Ho Choe, https://orcid.org/0000-0001-9899-9120

Ben Kang, https://orcid.org/0000-0002-8516-9803

\section{References}

1. Rosen MJ, Dhawan A, Saeed SA. Inflammatory bowel disease in children and adolescents. JAMA Pediatr 2015;169:1053-60.

2. Benchimol EI, Fortinsky KJ, Gozdyra P, Van den Heuvel M, Van Limbergen J, Griffiths AM. Epidemiology of pediatric inflammatory bowel disease: a systematic review of international trends. Inflamm Bowel Dis 2011;17:423-39.

3. Hong SJ, Cho SM, Choe BH, Jang HJ, Choi KH, Kang B, et al. Characteristics and incidence trends for pediatric inflammatory bowel disease in Daegu-Kyungpook Province in Korea: a multi-center study.J Korean Med Sci 2018;33:e132.

4. Kang B, Kim JE, Jung JH, Choe JY, Kim MJ, Choe YH, et al. Korean children and adolescents with Crohn's disease are more likely to present with perianal fistulizing disease at diagnosis compared to their European counterparts. Pediatr Gastroenterol Hepatol Nutr 2020;23:49-62.

5. Kang B, Choi SY, Kim HS, Kim K, Lee YM, Choe YH. Mucosal healing in paediatric patients with moderate-to-severe luminal Crohn's disease under combined immunosuppression: escalation versus early treatment.J Crohns Colitis 2016;10:1279-86.

6. Kang B, Choe YH. Early biologic treatment in pediatric Crohn's disease: catching the therapeutic window of opportunity in early disease by treat-to-target. Pediatr Gastroenterol Hepatol Nutr 2018;21:1-11.

7. Ruemmele FM, Veres G, Kolho KL, Griffiths A, Levine A, Escher JC, et al. Consensus guidelines of ECCO/ESPGHAN on the medical management of pediatric Crohn's disease. J Crohns Colitis 2014;8:1179-207.

8. Kim JH, Cheon JH, Kim WH. The frequency and the course of the adverse effects of azathioprine/6-mercaptopurine treatment in patients with inflammatory bowel disease. Korean J Gastro- 
enterol 2008;51:291-7.

9. Chun JY, Kang B, Lee YM, Lee SY, Kim MJ, Choe YH. Adverse events associated with azathioprine treatment in korean pediatric inflammatory bowel disease patients. Pediatr Gastroenterol Hepatol Nutr 2013;16:171-7.

10. Yang SK, Hong M, Baek J, Choi H, Zhao W, Jung Y, et al. A common missense variant in NUDT15 confers susceptibility to thiopurine-induced leukopenia. Nat Genet 2014;46:1017-20.

11. Lee YJ, Hwang EH, Park JH, Shin JH, Kang B, Kim SY. NUDT15 variant is the most common variant associated with thiopurine-induced early leukopenia and alopecia in Korean pediatric patients with Crohn's disease. Eur J Gastroenterol Hepatol 2016;28:475-8.

12. Kim HY, Lee SH, Lee MN, Kim JW, Kim YH, Kim MJ, et al. Complete sequence-based screening of TPMT variants in the Korean population. Pharmacogenet Genomics 2015;25:143-6.

13. Lee KM, Kim YS, Seo GS, Kim TO, Yang SK; IBD Study Group of the Korean Association for the Study of Intestinal Diseases. Use of thiopurines in inflammatory bowel disease: a consensus statement by the Korean Association for the Study of Intestinal Diseases (KASID). Intest Res 2015;13:193-207.

14. Asada A, Nishida A, Shioya M, Imaeda H, Inatomi O, Bamba S, et al. NUDT15 R139C-related thiopurine leukocytopenia is mediated by 6-thioguanine nucleotide-independent mechanism in Japanese patients with inflammatory bowel disease. J Gastroenterol 2016;51:22-9.
15. Zhu X, Wang XD, Chao K, Zhi M, Zheng H, Ruan HL, et al. NUDT15 polymorphisms are better than thiopurine S-methyltransferase as predictor of risk for thiopurine-induced leukopenia in Chinese patients with Crohn's disease. Aliment Pharmacol Ther 2016;44:967-75.

16. Collie-Duguid ES, Pritchard SC, Powrie RH, Sludden J, Collier DA, Li T, et al. The frequency and distribution of thiopurine methyltransferase alleles in Caucasian and Asian populations. Pharmacogenetics 1999;9:37-42.

17. Yang JJ, Landier W, Yang W, Liu C, Hageman L, Cheng C, et al. Inherited NUDT15 variant is a genetic determinant of mercaptopurine intolerance in children with acute lymphoblastic leukemia. J Clin Oncol 2015;33:1235-42.

18. Kim HT, Choi R, Won HH, Choe YH, Kang B, Lee K, et al. NUDT15 genotype distributions in the Korean population. Pharmacogenet Genomics 2017;27:197-200.

19. Relling MV, Schwab M, Whirl-Carrillo M, Suarez-Kurtz G, Pui $\mathrm{CH}$, Stein CM, et al. Clinical pharmacogenetics implementation consortium guideline for thiopurine dosing based on TPMT and NUDT15 genotypes: 2018 update. Clin Pharmacol Ther 2019;105:1095-105.

20. Kakuta Y, Naito T, Onodera M, Kuroha M, Kimura T, Shiga H, et al. NUDT15 R139C causes thiopurine-induced early severe hair loss and leukopenia in Japanese patients with IBD. Pharmacogenomics J 2016;16:280-5. 